\title{
Students' Needs Concerning Smoking Prevention: A Qualitative Comparative Analysis Among Public, Private, and Islamic School
}

\author{
Laili Rahayuwati1 ${ }^{1, *}$, Eleanor C. Castillo ${ }^{2}$ \\ ${ }^{1}$ Faculty of Nursing, Universitas Padjadjaran, Indonesia \\ ${ }^{2}$ College of Public Health, University of the Philippines Manila, Philippines
}

Received October 20, 2019; Revised January 10, 2020; Accepted January 17, 2020

Copyright $\odot 2020$ by authors, all rights reserved. Authors agree that this article remains permanently open access under the terms of the Creative Commons Attribution License 4.0 International License

\begin{abstract}
Smoking among adolescents is associated with additional health and social problems. This research was qualitative research and was used for exploring and describing the needs of smoking education program and strategies. The sampling technique of "snowball sampling" for male and female students, who are active smokers and non-smokers from different schools: Public, Private, and Islamic School; while the number of participants were 25 participants for interview and 30 participants for FGD. The qualitative data was analysed using thematic analysis. The qualitative results showed there were some factors contribute to smoking habits and the tailor-made of specific health education strategies should be implemented. It can be concluded that policy factors determine the success of health promotion and education. On one hand, schools are under the Department of Education and Culture, while community activities are referred to the Department of Home Affair, Department of Religious Affair. Therefore, improved cooperation between these two relevant departments is needed to regulate smoking and enhance the implementation of smoking prevention policies in schools.
\end{abstract}

Keywords School-based Intervention, Smoking Prevention, Adolescents

\section{Introduction}

Currently, in Indonesia, approximately 215 billion cigarettes are consumed every year by 57 million smokers. In 2010 , there are $34.4 \%$ of people aged above 10 years who smoked, with a higher prevalence in rural areas (36.6 percent), compared to $31.7 \%$ in urban areas, increasing from 31.5 in 2001.
Furthermore, the number of adolescents smoking: among the youth (age 13-15), 12\% currently smoke cigarettes (boys 24\%; girls 2\%); more than $78 \%$ of smokers start before age 19, one-third of whom reported trying their first cigarette before the age of 10 [1].

Additionally, in Indonesia, smoking exterminates at least 200,000 people each year. Secondly, more than 97 million Indonesian non-smokers are regularly exposed to secondhand smoke. Furthermore, $81 \%$ of youth (age 13-15) are exposed to secondhand smoke in public places, while $65 \%$ are exposed to secondhand smoke at home [2, $3]$.

As mentioned earlier, smoking poses a dilemma in Indonesia. On one side, it affects public health and contributes to a reduction in the quality of life of the people. On the other, it is beneficial to the government because the excise taxes contribute to fund public health programs. This act has been strengthened by the power of local and multinational tobacco companies on this archipelago of some 245 million people. For instance, most of the musical and art concerts for teens are sponsored by tobacco companies. Moreover, in 2008, PMI (Philip Morris International), the owner of PT. HM Sampurna (name of Indonesian tobacco company), became the market leader capturing 30 percent of the cigarette market share.

This study, thus, aims at exploring the students' needs and the supporting factors for health education strategy. This study is important for several reasons. Firstly, the study provided relevant information to the stakeholders about what factors influenced smoking initiation among a school's adolescents which, in turn, became the basis for smoking prevention plans. Secondly, it identified the gaps existing between the perspectives of adolescents, adults, and relevant stakeholders, especially in relation to smoking. Furthermore, the study responded to an urgent 
need for a "stop smoking initiation" policy and "stop smoking practices" initiatives which were better tailored to the realities of an increasing number of cigarette-smoking adolescents in schools. Therefore, this study focused to determine methods were needed to identify the variables that might later be tested quantitatively to determine the effects of the health education package.

The objective study were: 1) To explore the students' expectations and aspirations concerning the health education package (method, material, place, time) on smoking behavior prevention; 2) To describe the social environment and policy factors regarding school-based intervention for smoking behavior prevention.

Significance of the study: 1) The study responded to an urgent need for a "stop smoking initiation" policy and "stop smoking practices" initiatives which were better tailored to the realities of an increasing number of cigarette-smoking adolescents in schools. Specifically, the health education package in this study was developed through literature review, observation, in-depth interview and focus group discussion by considering the characteristics of the students and the school, the social and cultural backgrounds of West Java, and the policy environment of the schools. 2) In addition, this study provided the relevant stakeholders (teachers, parents), all those responsible for a "stop smoking initiation" and "stop smoking practice", with information for enhancing the positive health behavior of students within the school system.

\section{Literature Review}

Youth Health Survey Indonesia (2010) demonstrated almost $55 \%$ of adolescents in between $15-24$ year of age (52\% males and $3 \%$ females) were smokers. Among the current smokers between 30-34 years old, the survey showed that $66 \%$ were initiated into smoking at a young age.

There are several characteristics and risks associated with smoking behavior and with quitting and not quitting. Many of these factors are applicable not only to adolescents but also to all individuals.

In this study, the possible factors or independent variables which will affect the prevention of smoking behavior are: (1) internal factors, specifically the behavioral factors such as knowledge, attitude, and self-efficacy; (2) external factors such as social relationships and the environment (school system, teachers, peers, parents), including policy conditions at the country level and local regulations; (3) demographic factors of students (age, gender, ethnicity, social economic status of the family); and, (4) health education program. Furthermore, a dependent variable in this study is the smoking practice, in terms of which this study will concern itself with the intention to quit, the actual participants who quit smoking, and the commitment not to initiate smoking.

Many countries prohibit the marketing of tobacco use, but it is still allowed under Indonesia's laws. Global Youth Tobacco Survey (GYTS) stated that $73-80 \%$ adolescents who participated in the survey were exposed to some kind of cigarette advertisement through various media. The most used media for smoking advertisement are sports activities, other adolescents' activities, and billboards $(80 \%)$. In Jakarta, particularly, $92.4 \%$ of adolescents see the billboard advertisements, $88.7 \%$ watch the TV advertisements, and even more (92.4\%) see the advertisements during sports activities and adolescents' events. On average, $11 \%$ of adolescents surveyed globally have been offered cigarettes by cigarette companies, but in Jakarta, the percentage is actually higher (13.2\%). Moreover, because $70 \%$ of smokers in Indonesia began their smoking habit before they were 19 years of age, the cigarette industries aggressively target adolescents both directly and indirectly. All large tobacco companies in Indonesia are sponsoring sports activities, adolescent activities, and musical concerts, creating an environment to promote consumption of tobacco products as part of the social norm, especially among children and adolescents. Thus, Indonesian children are very much influenced by advertisements that feature tobacco use. Rapid increase in tobacco consumption among the youth occurred between 1995 and 2001[4].

It should be reminded that Indonesia is the most populous Muslim country in the world. Based on the Koran, the holy reference for Muslims, it is forbidden to consume food or any other substances which bring harm for the human body. Nevertheless, in Indonesia, smoking remains a controversial behavior, and several "ulama" (Muslim clerics) discussed whether smoking is an allowable habit or not. Forty-seven percent $(47 \%)$ of 'ulama' saw smoking as "haraam" (absolutely forbidden) and 26 percent saw it as "makruh" (strongly discouraged), while rest gave no opinions. Also, among "imams" (Muslim leaders) and employees of Islamic foundations, there was a difference of opinion on this matter which reflect various ethnic backgrounds (Indonesian, Arab, India, and Pakistani). Even though religious teachings alone will not have much effect on the smoking rate, by integrating them with behavioral and health and medical approaches.

They may have the potential to guide smoking cessation activities among Muslim smokers, especially during the month of Ramadan (during which smoking is prohibited) [5]. The strategy of using religious persuasions will be incorporated in this research for developing the material of health education program for smoking prevention among adolescents, especially in Islamic schools. 


\section{Materials and Method}

The descriptive qualitative study provided basic information for developing a health education package in a school setting. For the qualitative study phase, non-probability sampling method was used, further the basis for the selection of respondents was based on characteristics of individuals, in which they reflected the diversity.

Based on the qualitative study literature, the number of participants (male and female students) for interview were 25 participants. Also for FGDs (Focus Group Discussion), the participants were 30 , which were divided into three groups. Other data were obtained from informants which included schools' principals, teachers, and school counselors, the data was collected through interviews with teachers and parents.

The qualitative data were collected using in-depth interviews and a focus group discussion (FGD) conducted among the students in three junior high schools in Indonesia. Two schools were public, while the other was an Islamic private junior high school. The in-depth interviews were conducted with 25 students, while the three FGDs held consisted of 9, 10, and 11 students per FGD.

For the thematic analysis, the researcher followed step-by-step guidelines. These guidelines were: (1) familiarizing with the data; (2) generating initial codes; (3) reading through each transcript to immerse in the data; (4) reviewing themes; (5) defining and naming themes; and, (6) producing the report. For this qualitative study, the researcher merged findings with procedure. This method allowed the researcher to make generalizations about the cases.

\section{Results and Discussion}

The characteristics of the respondents were: the age ranging from 11 to 13 years old numbered to 37 students $(67.5 \%)$, while that of 14 to 17 years old numbered 18 students $(32.5 \%)$. There were 39 male students $(70 \%)$ and 26 female students $(40 \%)$, the majority of whom $(98 \%)$ belonged to Muslim religious group, while only $2 \%$ belonged to the Roman Catholic and Christian Protestant religious groups. Ethnically, most $(91.5 \%)$ belonged to Sundanese group, while the rest to Javanese and Bataknese (8.5\%).

The Sundanese ethnic group, who resides in West Java Province, is the second largest ethnic group in Indonesia. Although inhabiting the same island with Javanese ethnic group, the Sundanese consider the territory they live in as a culturally distinct territory and refer to it as Sunda (majority ethnic name in West Java). The Sundanese culture also retains specific characteristics, distinguishing it from other cultures with which it shares. The term Sunda is indeed used in connection with its people; therefore, an urang Sunda is a person who claims this denomination for himself or herself and is acknowledged by other people as being a Sundanese, a definition based on both hereditary and socio-cultural aspects. In addition, a person or a group of people is called Sundanese when raised in a Sundanese socio-cultural environment and fully comprehend and live in accordance with its norms and cultural values. In this matter, the place of residence, the socio-cultural life, and the parents' attitudes, values and behavior are considered important.

Based on the data collection and qualitative analysis on students' expectations and aspirations concerning health education and factors regarding smoking prevention, there were three predominant themes which emerged with regard to students' perceptions on smoking and its prevention: 1) prefered health education strategies in terms of method, materials, place and time ; 2) individual characteristics, reasons, and impact of smoking; 3) family, social, and school and policy factors contributing to smoking habits--barriers and supporting systems.

\subsection{Students' Expectations and Aspirations Concerning Health Education Package}

The objective of the intervention program was to enhance the students' knowledge, self-efficacy, and personal behavior, aside from encouraging them to quit smoking. Therefore, the health strategies necessary to meet the needs of the adolescents have become, indeed, more challenging. The health education strategies need to consider the demographic pattern of students and the advancement of technology which can contribute to the effectiveness of approaches to adolescents.

In addition, the information concerning cultural values, beliefs, behaviors, and practices of the students are very important. It is helpful to know their reference framework and their needs regarding smoking behavior and its prevention. Consequently, based on the students' demand for an educational program, health educators were challenged to meet the changing needs of the students as participants of the program in new and different ways.

\subsection{Preferred Health Education Methods}

The planning of the program was guided by a comprehensive understanding of the health educators as a team and the strengths and needs of the student learning process. The preferred learning process of the students was the active style ("student-centered") rather than the passive style ("health educator-centered"). Some participants perceived the health education processes and strategies as fun, while some perceived them as aggressive (watching attractive video and having discussions), competitive (games), or threatening (role-playing, or small group activity). Several students said, "We prefered the health education strategies in the interesting and attractive way, involving games or role play." 
However, this study also found that not all students were aware of the objectives of the health education program. They were enthusiastic for being participants in the health education program because the atmosphere was more informal, utilizing interesting audio visual materials.

From their statements, the study recognized various barriers to learning such as the mismatch of expectations between the health educators and students. Based on the transcription, the findings showed that most students preferred an interactive method of health education such as visual teaching materials (i.e., videos, games), ice breakers, and dramatization (such as role play, comedy). Some students recommended that, in order for seminars or class teaching modules to be effective, students be given more preparation time and a more convenient place for those activities.

The students stated that they needed to have adequate information which could be carried out through education. The findings also revealed that majority of the students have heard various information about smoking, but they were confused as to their accuracy. As they furthered, "In fact we have heard a lot of information regarding smoking both from peers and media, but we are not convinced if the information can affect our smoking habits."

The students also gave the impression that they were confused as to why they became smokers. It was interesting to note that they were clearly upset about being smokers, and thus, they inquired about ways and searched for training on how to quit smoking.

Some students explicitly expressed that they needed specific health education related to smoking which especially targets the youth. This study discovered that they strongly felt the need to acquire education materials, such as: the history of cigarette and smoking, the cigarette industry, the effect and impact of smoking on health, the psychological and religious factors affecting smoking behavior, the reasons and motivations for smoking habits, and the characteristics of smokers.

As indicated in the Introduction, the schools were chosen for the reason that they were ideal places to develop and to continuously evaluate the intervention program of smoking prevention for adolescents attending formal education. Assuming that the students spent 5-7 hours at school every day, they would be exposed to every intervention program in the institutions. Some students also recommended the convenient time (during school-time) and a more convenient place for these activities (either outdoor or indoor).

\subsection{Individual Characteristics Related to the Reasons and Impact of Smoking Behavior}

The study from all schools revealed that more than half of the students have intentions to smoke.This intention of whether they smoke or not was, in fact, due to various reasons.Among the students from the Islamic school, the reasons for not smoking were mostly related to their religious beliefs. According to the data gathered, most of the students said that there is a rule in the religion which prevents them from being smokers. Some students referred to a Koranic verse: "Muslim should avoid harmful goods and negative behavior activity as The Koran guidance from Al A'raf: 17" as their basis for not smoking. However, their motivation for smoking or not is also relative on the faith of that person.

Some students argued that they needed to sacrifice a lot to smoke, namely, that not only is smoking money-consuming, but also lurks a negative effect to the environment and other people through air pollution. If one would stop smoking, he/she could save money for the future spending as well as minimize imminent health care costs. In addition, some students stated that they did not smoke because of its unpleasant taste.

Some students refused to smoke because their friends did not feel comfortable with the smoke and smell of the cigarette, and were, consequently, afraid that they would be avoided or ousted from their social circle. As one student explained, "I avoid smoking because I saw some friends casting out the smoker students."

On the contrary, those who smoke have various reasons which motivated them to do so. Exposure to tobacco advertisement was cited as one of the main reasons. Accordingly, based on the advertisement, the students only needed $\mathrm{Rp} 1,000.00$ (one thousand rupiahs, equivalent to 0,14 US dollar) to buy one cigarette, while their allowance or pocket money were $\mathrm{Rp} 15,000,00$ (fifteen thousand rupiahs, equivalent to 1 US dollar). Thus, they had enough money to spend for cigarettes. A few students said that many of the cigarette advertisement designs used in posters, pictures, and electronic mass media enticed them highly and motivated them to try and taste cigarette-smoking. In addition, some concerts sell their tickets inclusive of a free pack of cigarettes as part of the concert sponsorship and cigarette advertisement. While watching or enjoying the music during concerts, people are allowed to smoke.

When the financial factor was not sufficient as a reason, some students said that they smoked because their parents had a small cigarette shop or were ambulant cigarette vendors. Other students were able to buy cigarettes because of money from friends - either loaned or given as a gift.

Results also revealed that smoking was also closely associated with having a macho image and its perceived benefit. As some students stated, "a smoker male student looks more mature, stronger, an attractive leader in the eyes of the opposite sex, and being cool". Others believed that when they smoke, especially when they copy the style of a popular public figure, they get positive association in the minds of other people: "one looks like a popular public figure by imitating him/her smoking a cigarette."

However, this perception is reversed when it came to 
female students who smoked. A female smoker is commonly associated with a negative stereotype. An anonymous respondent stated: "The female smoker is a bad woman and commonly working in the sex industry."

In spite of various motivations for students to smoke, most of the smoker student respondents did not perceive smoking as an addiction. In fact, they even saw it as a source of pleasure. It was also common among the smokers to see the activity as a coping mechanism. Some male students stated that smoking helped them deal with psychological problems and stress. Smoking also helped them manage overloaded student tasks, maintain private and exclusive relationship with girlfriends.

It was also interesting to find that they have tried smoking to know its taste.Although nauseous in the first try, many, unfortunately, continued to smoke thereafter. When it came to cigarette taste as a factor, some students liked its flavor, especially those that taste like an apple or an orange.

\subsection{Perceived Impact of Smoking Behavior}

Regarding the impact of smoking behavior, most students answered that it had some effects on the body, such as: "being physically thin, having dark color of lips, and regular cough." Despite the knowledge in the memorization stage, little did they know the real hazards that smoking can impose on their health. They could not enumerate what kind of harmful effects the cigarette's substances have.

Some students said that they only learned the diseases from the warning labels on the cigarette packs. The warning labels stated: "Smoking can cause diseases of the lung, pneumonia, renal disease, cancer, cardiovascular diseases, impotence, harm to the pregnant women, and infertility." From the in-depth interviews, only a few students explained correctly the kind of diseases mentioned on the cigarette packs. In addition, only a few answered the diseases caused by smoking correctly.

From knowing the negative effects of smoking, some students analyzed the risks inflicted by it, whether among the adolescents or adults, and categorized the health difference between the smoker and non-smoker in this way: "the non-smokers look healthier in comparison to the smokers; thus, if I could have a choice, I would prefer to be a non-smoker."

In fact, some students were also well aware of the cigarette warnings against smoking for those below 17 years old. However, they perceived that they will still be allowed to smoke upon reaching 17 years old.

\subsection{Family, Social, School, and Policy Factors Affecting Smoking Behavior}

The nuclear family (involving father, mother, brothers, and sisters) and the extended family (involving grandfather, grandmother, uncles, aunts, and cousins) influence one's smoking behavior. As one student stated: "Since five years ago, I have seen my father and mother possessing a smoking habit, either at home or outside". From another student: "My father was a drug user. And I saw smoking was a common practice back then. It was far better to smoke than to use drugs." Another student, knowing that most of his family members were smokers, assumed that smoking behavior was a hereditary trait.

Obviously, some students said that they become smokers because of permissive family environment. Particularly, most of the student respondents lived in a household with member of the smoking, e.g. the father, mother, siblings, or in-laws. Even, some parents allowed their children to be smokers, and they would rather have them as smokers than alcohol drinkers. As students stated that their parents said: "It is better to be smoker than alcohol drinkers". In addition, some students reasoned that they helped their parents increase family income, and, in return, the parents would allow them to smoke.

Based on the students' experiences, some students admitted that, since they started to be regular smokers, they bought cigarettes together with their fathers. In this case, the smoking habit of the students began at home within the family environment. In addition, some students felt free to smoke since they receive the family's tolerance, even support. The students were made to understand that it is permissible for them to smoke when they are grown up, as their parents were often heard to say, "If you grew up and already had salary or income, you would be free to smoke".

For the non-smokers, it was noted that there were several barriers which either prevented or motivated the person not to smoke. Some students admitted, "I would not smoke because of an experience of a family member who got sick or suffered from diseases related to cigarette." Other students explained that they did not smoke because they did not have smoking family member/s.

With regard to the family's role in smoking prevention, few students stated that their parents would be angry had they known that their children smoked either at school or somewhere else. Other students believed that it was important to build a good family rapport between parents and children in order to prevent smoking behavior among students.

Among the social factors that influenced the students' smoking behavior, it was peer pressure that was first identified. The smoker students viewed their smoking peers as "cool" or "great", while the non-smokers were "out-of-date". Cigarettes even became part of their social etiquette: offering a cigarette became a customary practice whenever one met his/her friends or peers. Others stated that the younger students were usually offered a cigarette by their older friends or their seniors in the school. Hence, the younger ones were encouraged to smoke as part of 
friendship. The students admitted that their smoking habit developed gradually. After the first attempt, they will try another time with one cigarette or more, until finally it becomes a habit with finishing one pack or more a day.

To prevent smoking, some students thought that peer prevention support could be effective, i.e. a student could remind his/her friends not to smoke. They also believed that neighbourhood environment also contributed to the development of smoking habits among students, particularly in the areas where there were shops or vendors selling cigarette. In other words, there were free markets for cigarette trading (ranging from small to big groceries) which gave opportunity for the students to acquire cigarette easily and to develop smoking habits. Notably, when the vendors asked them for whom they are buying the cigarette, the students always answered "for their fathers".

Moreover, there is no restriction or negative reinforcement from the social environment concerning smoking behavior: the society does not discriminate or stigmatize against the smoker students.

In the school environment, this study found that students were free to smoke when there was no smoking prohibition in the school. Even a teacher once told them, "Feel free to smoke because smoking is a human right, but the smokers do not wear the X of a junior high school uniform."

When the school rules included the smoking prohibition, some students smoked in the toilets. However, sometimes, when some teachers found students smoking cigarettes in the $\mathrm{CR}$, they did not give any penalty except an advice. A few students noticed this and said that "the teacher did not punish us; he/she only gave reminders 'not to smoke'.". The study also revealed that some students began their smoking habits when they were in the Primary School. In addition, in some Junior High Schools, even some personnel in the canteens sold cigarettes secretly to the students.

The study also found that the students were more concerned with their present condition only, and that they were more having influences with the advertisements. As a few students explained that "here were a lot of smoking and cigarette advertisements through big posters and banners surrounding junior high school environment". Several even believed that "it will be alright to start smoking at the age of 17 years old". Moreover, other students perceived that mild cigarettes have filters and contained low nicotine and thus are safer for their health.

There is, in fact, school policy system for preventing smoking in schools such as the "No Smoking" policy, and it was found that only the Islamic school strictly implements it. The two other schools in this study did not have a strict implementation on smoking prevention. In relation to school environment protection, the regulation of the public schools imposed a penalty of Rp 24,000.00 (twenty-four thousand rupiahs, equivalent to two US dollar) to any student caught smoking in the school compound/complex, some students stated that "despite the prohibiting regulations against smoking in the school, some students still smoked within the compound."

The health warnings on the cigarette packs throughout history: 1) until 1999, it contained: "Government Warning: Smoking Can Harm the Health"; 2) 1999-2001: "Government Warning: Smoking Can Cause Cancer, Heart Attack, Impotence, and Pregnancy and Fetal Disorders"; 3) 2002-2013: "Smoking Can Cause Cancer, Heart Attack, Impotence, and Pregnancy and Fetal Disorders"; 4) 2014: "Smoking Kills You." In addition, cigarette warnings nowadays include (for advertisement) the sign saying "18+" on the right-hand side.

In the middle of 2014, the Indonesian Government changed the policy regarding cigarette warnings. In addition to the common warning statements, the cigarette packs now features several pictures of diseases associated with smoking together with the statement: "Smoking kills you". Although this warning labels targets all people, this study revealed that there is no government policy strictly being implemented that will prevent people under 17 from smoking.

From these results, it was found that the perceived impact of smoking behavior on students is still at the shallow level of knowing or memorization. Moreover, there is no specific government policy which prevents smoking among under-17 students who are in school. Hence, the existence and application of a "No Smoking" policy depends on the school system.

Based on qualitative analysis, data on respondents' demographic characteristics similarly depict the condition of students in the Province of West Java. These data are comparisons in the scope of West Java province with the research situation. The number of Sundanese (population 78.3\%: participants 90\%); the number of Moslems (population 97\%: participants $97.25 \%$ ).

The qualitative results of this research show that some students prefer the following methods, which is, among others, students' expectation for interactive, fun and relaxing methods. In addition, students expect that the materials on health education be suitable for their needs and able to illuminate them regarding the basic knowledge of smoking, its advantages and disadvantages. This expectation basically implies that many students have not understood correctly and comprehensively about smoking and its effects. It is possible then that the smoking behavior they display since the first year of their secondary school is not based on proper understanding of smoking and its impacts. Although not all methods and media can be adapted in this research, the information regarding the various methods expected by students is invaluable and can be made input in increasing changes in health behaviors.

This phenomenon can also be observed in students' 
confusion about cigarette and smoking that makes them uncertain with the decision to make, and they eventually base their decision only on the knowledge about cigarette they gain from several media. The qualitative data further confirm this analysis that the environment, either family, peers, school, or policy system has a significant influence on students' smoking behavior.

The smoking age in Indonesia has been increasingly decreasing, where some data show that the age of first-time smokers of the population was in the range of $15-19$ years old (33.1\%) in 2007, and rose to $43.3 \%$ in 2010. The smoking age group of 10-14 years old in the same period also experienced an increase from $10.3 \%$ to 17.5\% (Global Youth Tobacco Survey, 2010). Meanwhile, in this research, some subjects were identified as first time smokers. Adolescents as the subjects of this research significantly require intervention because most of the adolescent students have not got addicted to smoking and their behaviors can possibly be changed. The primary prevention in this research was indeed aimed at a group of students who have not started smoking in order to avoid smoking habit. Meanwhile, for the first time smokers, secondary prevention was applied, namely in order to minimize the risks of smoking-related diseases and increase their knowledge about cigarette as well as providing them with guidelines to stop smoking.

\subsection{Social Characteristics and Media Effects}

Meanwhile, it cannot be denied that social media have a great influence in one's life. For Indonesian people, particularly the adolescents, social media is addictive, where there is no day without logging in into the media, and they cannot even get separated from the social media for 24 hours. Adolescent is the stage that covers the mental, emotional, social, and physical dimensions. In addition, adolescent is the stage of transition, as those who are at this stage have not reached adulthood, but no longer have the status of a child. Recent findings show that adolescents have high dependency on social media. Research conducted by higher education institutions in Indonesia in collaboration with yahoo shows that adolescents at the age of 15-19 years old dominated the internet users in Indonesia for $64 \%$. It has to be noted that mass media, including social media, have allowed for the coming of new cultural patterns, both negative and positive ones, and have begun to determine society's mindset as well as behaviors [6].

In reality, mass media are an outlet by which society's activities in general can be monitored. However, this use of mass media has not been effectively implemented in controlling cigarette, especially targeted at adolescents, as reflected in the Decree of the Minister of Health of 2013 concerning cigarette control. In fact, the media can actually implement this control by providing warning and other forms of social control employing persuasive activities. Social monitoring and control can indeed be done to prevent undesirable things. Likewise, reporting by mass media on the effects of smoking, especially on adolescents, can serve as prevention, guidance, and education in order for adolescents not to fall into unhealthy lifestyle [7].

A shift in patterns of behavior, in addition to being caused by the mass media, according to the Department of National Education (2004) can be a result of influences from peers, family members, schools, and community. Changes in behaviors, among others, can be observed in lifestyle, where one imitates a figure of an idol based on the information obtained from the media. A person will commonly imitate anything related to his or her idol, either in terms of dressing style, appearance, haircut, or speech style that can reflect his or her idol. This argument is supported by other research findings, such as the one by "There were a lot of smoking and cigarette advertisements through big posters and banners surrounding junior high school environment", and "one looks like a popular public figure by imitating him/her smoking a cigarette."

With regard to cigarette advertisements targeting those who are under 17 years old, as put forward in a video released by an anti-cigarette non-government organization in Bandung, even though the cigarette producers do not include those who are under 17 years old as their ads' targets, the approaches they employed, such as posting banners of cigarette ads in the vicinity of schools, supporting music concert very well-liked by adolescents while implicitly advertising cigarettes, and including young actors and actress in the cigarette campaigns (mostly implicitly), show that Indonesian adolescents are the potential target for cigarette consumers because in terms of number, they make up a large percentage of the whole population.

Specifically, there are several factors that may affect smoking behavior changes among the students in this research, such as explained below: The first factor, Individually and institutionally, religion can be said to have an influence on smoking habit. This concern with religion is not solely about the kind of religion students embrace, since the majority of students are Muslim, but more about the quality of their understanding and how they practice their religion. The better students' understanding of religion and its rules is, in this case Islam, the more unlikely they will smoke. This argument is supported by some of their statements which indicate whether or not they consider religion in their decision making.

Nevertheless, qualitative data also show that there are contradictory data between smoker and non-smoker. For the non-smoker group, their decision not to be a smoker is based on their perception that cigarette has a bad taste when they first tried it. On the other hand, the smoking students stated that their addiction to smoking is caused 
by the good taste of cigarette. It can be inferred then that there is a tendency that students who do not like smoking have had bad initial experience with it. However, those who attempt to smoke a couple of times or repeatedly will eventually like the taste of cigarette. This is in line with adult's experience, in which they have difficulties in quitting smoking due to the addictive substances contained in cigarettes. Several resources indeed mention that nicotine is one of the substances contained in cigarettes that can cause addiction $[8,9]$.

The view regarding smokers also indicates a double standard. For instance, there is a tendency to attach the image of macho and attractive to male smokers; on the other hand, female smokers are generally associated with something negative, such as the profession of a prostitute.

In general, students are very much informed about the impacts of cigarettes that are observable on skin color, coughing, and other health symptoms. However, they do not consider the effects of cigarettes and the danger caused by its addiction. This phenomenon to some extent reflects adolescent's characteristic that is more likely to be concerned with the here and now and have no clear plan for their future. Brighton [10] explained that in the transition period, adolescents want to search for their self-identity and freedom for the here and now; therefore, they have an inclination to experiment with drugs, alcohol, or sex.

The second factor is social and physical environment where the students interact. The social environment includes: family, household, peer interaction, relationship with the community, and the like. The individual factors is strengthened by the permissiveness of family, peer support for smoking, a lack of social concern with adolescents' smoking behavior, the influence of cigarette ads in the media (the large number of cigarette products advertised), the spread of smoking habit at home (smoking tends to be "inherited" and is more preferable than drinking and using drugs), school environment that is exposed to cigarette ads, and the weak government policy in protecting adolescents against the danger of smoking.

With the advancement in information technology, parents and teachers can no longer restrict the students' environment, which has become increasingly widened and global. Moreover, recently the information technology has developed so rapidly that school teachers encounter challenges in students' unhealthy and even hazardous behaviors, such as smoking; consuming alcoholic beverages and similarly drunk-inducing drinks, narcotics, psychotropic and other addictive substances; and practicing unhealthy sexual behaviors $[11,12]$. Consequently, student development becomes more difficult to be predicted or monitored both by teachers and parents.

With regard to the family environment, the qualitative data found when there was family or social environment that "tolerates" smoking behavior, especially upon having received own salary, increases the tendency that the young who stayed within this environment would become smokers.

In addition, the students' behaviors are determined by mutual support from the environment. On the other hand, unsupportive environment, such as broken home family, an environment with moral decadence, and smoking family members, will easily influence students to smoke $[13,14,15]$.

Despite all the different factors and environmental reasons contributing to the smoking behavior of the students, it is interesting to note that the school policy of smoking prohibition did influence students' behavior on smoking. The school regulation against smoking could influence personal attitudes of the students towards smoking. Based on the students' information and observations, smoking seems more acceptable in the public schools than in the Islamic school. The schools without a strict "No Smoking" regulation could be more lenient to students who try smoking within their areas and this, more often than not, lead students to develop a smoking habit.

In terms of policy factors, the strength of the policy system, either in the government or school, are very important. The study indicated that students were more at risk into becoming smokers if they are studying in a school without a "No Smoking" regulation. Even a less vulnerable student could be prone to try smoking given a school environment which does not strictly oppose it. These findings provide empirical evidence to support the current theoretical literature that the characteristic of the school where a student attended may become a risk factor to personal smoking behavior.

Nevertheless, this study found the weakness of health system and strategies to reduce the smoking prevalence. Specifically, there are two types of policy factors. The first is for government policy to regulate the community activities under the Department of Home Affairs, while the second is school policy to regulate the school activities under the Department of Education and Culture. Under government policy in those district areas, there were no specific regulation in terms of smoking prevention, there is still no restriction of tobacco advertising surrounding school areas and affordability of tobacco (lower cost of tobacco) and availability (can be found in every shop and mini markets).

The third factor is socio-cultural values, marked by the Sundanese culture that strongly upholds collectivity and solidarity. This cultural value will be reflected in the students' daily life. From the observation, it is clear that Sundanese families prefer to live side by side with their clan; for instance, a house is frequently found to be occupied by an extended family, namely, parents, children, and other family members, such as grandmother, aunt, uncle, and nephews/nieces. In addition, in terms of residential location, Sundanese people tend to prefer to 
live closely to each other. It is frequently the case that several houses are found in one location (3-5 houses), whose occupants are members of an extended family. It is the socio-cultural factor that allows for changes or formation of an individual's behavior.

\section{Conclusions}

Students preferred a health education intervention which uses an interactive method of teaching such as visual teaching materials, ice breakers, and dramatization. Some students recommended that, for seminars or class teaching modules, finding a convenient place for these activities is very welcome. Furthermore, the two tailor-made interactive intervention strategies were built into the programs to prevent smoking.

Based on the findings, some recommendations for the effectiveness of the program based on the special characteristics of students in West Java Schools, is to develop a health education plan that takes into account the students' age, their development level, background, the socio-cultural norms around them, school goals, and students' needs. In order to determine the educational content to be given in a certain school, other factors have to also be considered, such as the beliefs and values of the society, school members' attitude and aspiration, and the opinion of parents and the prominent members of the society.

\section{Acknowledgments}

I would like to thank the Ministry of Research Technology and Higher Education of Indonesia which provided the opportunity and financial support for this study. I wish to acknowledge former Rector of Universitas Padjadjaran, Prof. Ganjar Kurnia, PhD, and Rector Universitas Padjadjaran, Prof. Dr. Tri Hanggono Achmad for great support to my study.

\section{REFERENCES}

[1] Global Youth Tobacco Survey, Indonesia - National 2006. Atlanta: Centers for Disease Control and Prevention. Available from: http://www.searo.who.int/tobacco/data/ino_rtc_reports/en/, 2014.

[2] Global Youth Tobacco Survey, Indonesia - National 2006. Atlanta: Centers for Disease Control and Prevention. Available from: http://apps.nccd.cdc.gov/OSH_GTSS/default/Default.aspx. on 21 July 2009, 2006.

[3] World Tobacco Asia, Trade shows. Available from: https://www.ntradeshows.com/world-tobacco-asia/. Jakarta,
2012.

[4] S. Barber, T. Aditama, J. Pradono, K. Rahman, C. Warren, N. R. Jones, S. Asma, J. Lee, Global Youth Tobacco Survey (GYTS) in Indonesia. Preventive Medicine, Vol. 47, S11-S14, 2008

[5] N. Nawi, L. Weinehall, A. Ohman, 'If I don't smoke, I'm not a real man'-Indonesian Teenage Boys' views About Smoking. Health Education. Research, Vol. 22, No. 6, 794-804, 2007

[6] Y. Jatnika, Jangan Larang anak main gadget. Ministry of Education and Culture. Available from: http://sahabatkeluarga.kemdikbud.go.id/laman/index.php?r $=$ tpost $/$ view\&id $=809,2015$

[7] M. H. W, Selfhout, S. J. T. Branje., M. Delsing, T. F. M. Bogt, W. H. J. Meeu, Different types of Internet use, depression, and social anxiety: The role of perceived friendship quality, Journal Adolescence, 2009.

[8] Centers for Disease Control and Prevention, Fact Sheet: Smoking Cessation, Available from www.cdc.gov /tobacco/data_statistics/fact_sheets/ cessation/quitting/, 2010.

[9] Centers for Disease Control and Prevention, Fact Sheet: Health Effects of Cigarette Smoking. Available from http://www.cdc.gov/tobacco/data_statistics/fact_sheets/hea lth_effects/effects_cig_smoking/\#cance2015

[10] K. L. Brighton, Coming of age: The education and development of young adolescents, Westerville, $\mathrm{OH}$ : National Middle School Association

[11] P. Cordingley, M. Bell, C. Isham, D. Evans, A. Firth, What do Specialists do in CPD Programmes for which There is Evidence of Positive Outcomes for Pupils and Teachers? Report in: Research Evidence in Education Library. London: EPPI-Centre, Social Science Research Unit, Institute of Education, University of London, 2007

[12] H. C. Kelman, Social Influence and Personal Belief: A Theoretical and Experimental Approach to Study of Behavior Change. New York: John Wiley and Sons, 1959.

[13] Global Youth Tobacco Survey Collaborative Group, Tobacco Use among Youth: a Cross Country Comparison. Tobbaco Control, Vol. 11, 252-70, 2002

[14] Global Health Professional Survey (GHPS) Indonesia, Tobacco Cessation. SEARO, New Delhi, 2006.

[15] Global Tax News, Available from http://www.tax-news.com/, 2012 